\title{
Les stratégies de coping que les jeunes homosexuels mettent en place lors de la divulgation de leur homosexualité \\ Charbonnier Elodie et Graziani Pierluigi
}

\section{Résumé :}

La divulgation de son homosexualité est une des expériences les plus stressantes que les jeunes homosexuels rencontrent. Cette étude vise à mieux comprendre la manière dont les jeunes homosexuels y font face.

Cette recherche a été menée auprès de 200 jeunes se disant homosexuels, âgés de 16 à 25 ans (69 femmes, 131 hommes). Les stratégies de coping employées lors de l'annonce de leur homosexualité la plus stressante qu'ils ont rencontrée ont été évaluées, tout comme la préparation et l'initiative de l'annonce, la présence d'idéations suicidaires et de tentatives de suicide.

Dans notre échantillon, $38 \%$ des répondants ont pensé à mettre fin à leurs jours durant cette divulgation, $23 \%$ ont réalisé une tentative de suicide. Ces données illustrent la vulnérabilité des jeunes homosexuels lors de la divulgation de leur homosexualité. Les jeunes qui ont préparé cette annonce et qui n'ont pas eu d'idéations suicidaires ont évalué de manière positive cette situation et se sont tournés vers les autres. Alors que les jeunes homosexuels qui ont peu préparé leur divulgation et qui ont présenté des conduites ou des idéations suicidaires, ont eu tendance à se remettre en question, à se culpabiliser et à s'isoler.

Selon nous, une approche cognitive, comportementale et émotionnelle permettrait d'accompagner les jeunes homosexuels dans la préparation et la divulgation de leur homosexualité, et favoriserait la prévention du suicide.

Mots-clés : Stratégies de coping ; stress ; suicide ; homosexualité ;

\section{Title: The coping strategies that young homosexuals set up at the time of the disclosure of their homosexuality}

\begin{abstract}
Introduction: Disclosing homosexuality can be one of the most stressful experiences a young homosexual can go through. It can generate significant discomfort with the young homosexual exposing him/herself to the opinions of others and to possible rejection. Yet it also represents an important step in the construction of the homosexual identity. This study aims to better understand how young homosexuals cope with the stress experienced as a result of 'coming out'.
\end{abstract}

Methodology: This research was conducted with 200 young people who indicated being homosexual, aged between 16 to 25 years (69 women, 131 men). Coping strategies, planning, initiative and rehearsal of the stressful situation were evaluated, as well as the presence of suicidal ideation and veritable suicide attempts. Participants were instructed to answer based on the most stressful 'coming out' that they had yet experienced.

Results: The coping strategies used by participants in the disclosure of their homosexuality were diverse. Strategies aiming towards personal development or problem solving were mixed with avoidance, guilt and isolation tactics. In the current sample, the most stressful announcement took place on average at age 18 . For $81 \%$ of the participants, it involved at 
least one of their family members. Nearly half of respondents said they "somewhat" or "not at all" planned this announcement. The statistical analysis shows that given an increased preparation of the coming out, the greater the chances that the individual will use problemsolving strategies $(\mathrm{r}=0.39, \mathrm{p}<0.0001)$, personal development and positive revaluation of the situation $(\mathrm{r}=0.22, \mathrm{p}<0.002)$. During their most stressful announcement, $38 \%$ of respondents thought about ending their lives and $23 \%$ made a suicide attempt. These data illustrate the vulnerability of young people associated with the announcement of homosexuality. Respondents who had suicidal ideation or suicidal behavior did not use the same coping strategies as other participants. Suicidal youths, when compared with nonsuicidal youths, tended to use escape mechanisms and avoidance $(\mathrm{t}=2.7, \mathrm{p}=0.007)$, feeling isolation and guilt $(\mathrm{t}=12.2, \mathrm{p}<0.0001)$, and addictive behavior and/or risk-taking behavior $(\mathrm{t}=5.1, \mathrm{p}<0.0001)$.

Discussion: Despite the stress involved in coming out, many participants used personal development strategies. It illustrates the importance of this announcement in the construction of identity and in personal development. But for others, this announcement is a moment of suffering sometimes leading to suicidal behavior. For the latter, escape mechanisms, isolation and addictive behaviors, are often involved in announcing their homosexuality with rare use of personal development strategies. These contradictions reflect the complexity of this announcement, which may be responsible for some wellbeing as well as some suffering, depending on the cognitive assessment of the situation and on the reactions of those around. Given these results, it appears that appropriate support, like a cognitive, behavioral and emotional approach based on stress management programs, could help in preparing this announcement and may prevent suicidal behavior. The cognitive axis could be used to attempt at improving the preparation of this announcement, as well as dealing with the reactions and consequences that may result. Therapy centered on emotions could also be used, which would aim at identifying and evaluating feelings experiences by young homosexual, towards an acceptance of their emotional experience and reducing their desire for control. Developing and using roleplaying could provide additional support.

Key words: Coping; stress; suicide; homosexuality 


\section{Introduction}

Cette étude a pour objectif de mieux comprendre la manière dont les jeunes homosexuels font face au stress présent lors de la divulgation de leur homosexualité à leur entourage. Dévoiler son homosexualité à un tiers est souvent un moment difficile et stressant $[1,2]$ mais pas forcément un stresseur négatif [3]. En effet, cette divulgation constitue un temps important, qui peut favoriser la construction de l'identité et l'épanouissement personnel [4]. La construction de l'identité homosexuelle est composée de différentes étapes telles que l'annonce à soi-même, l'annonce à son entourage et l'annonce à sa famille [5]. Ces étapes font parties intégrantes du «processus de coming-out», qui peut déboucher sur un «processus de reconnaissance de soi », venant favoriser le développement personnel et le bien-être des jeunes homosexuels [6]. Dans le cadre de cette recherche, nous nous sommes intéressés à un moment particulier de ce « processus de coming-out », à savoir l'annonce à un tiers.

Les homosexuels représentent un des groupes d'individus les plus stressés [7]. Les réactions familiales ou encore les attitudes de la société sont des stresseurs importants pour les homosexuels [8,9], mais pas autant que la divulgation de leur homosexualité [10]. Les diverses expériences stressantes auxquelles les homosexuels sont confrontés ont été regroupées sous le terme «minority stress » [11]. Ce modèle rend compte de l'excès de stress ressenti par les individus appartenant à des catégories sociales stigmatisées. Selon ce modèle, il existerait un continuum entre des stresseurs distaux et des stresseurs proximaux. Un stresseur distal correspondant à des évènements extérieurs, objectifs, ne dépendant pas de l'individu. Les stresseurs proximaux sont plus subjectifs, ils sont liés aux caractéristiques de l'individu. Nous considérons que la divulgation de son homosexualité constitue à la fois un stresseur proximal et distal. Nous nous positionnons en accord avec le modèle transactionnel du stress de Lazarus et Folkman $[12,13]$ et nous considérons donc que le stress est dépendant de la qualité de la relation entre l'environnement et l'individu. Pour faire face à une confrontation stressante, les individus mettent en place des processus de coping, c'est-à-dire des stratégies visant à résoudre la situation stressante. Ces stratégies adaptatives, ces efforts cognitifs et comportementaux, ont pour but de faire face à une situation évaluée par le sujet comme dépassant ses limites $[12,14]$. Dans le cadre de cette étude nous chercherons à 
approfondir les stratégies de coping que les jeunes homosexuels mettent en place lors de l'annonce de leur homosexualité.

Les études Nord-Américaines menées auprès de jeunes homosexuels démontrent qu'ils présentent un taux de tentatives de suicide, d'idéations suicidaires, d'épisodes dépressifs et de suicides réussis, plus marqué que les hétérosexuels [15-20]. Les hommes homosexuels ont entre 1,5 et 3 fois plus de risque d'avoir des idées suicidaires et sont entre 1,5 et 7 fois plus susceptibles de tenter de suicider que leurs pairs hétérosexuels [21]. La même tendance est observée en France pour les hommes, mais les résultats concernant les femmes sont contradictoires. Pour certains auteurs, les lesbiennes n'auraient pas plus de conduites ou idéations suicidaires que les femmes hétérosexuelles [22], pour d'autres, elles auraient deux fois plus de risque de réaliser une tentative de suicide [23]. Ces divergences peuvent être expliquées par des différences méthodologiques. La première étude se base sur les comportements sexuels au cours des douze derniers mois, elle exclut donc les personnes inactives sexuellement dans l'année. À l'inverse, la seconde tient compte des comportements sexuels dans la vie entière. Les données françaises méritent donc d'être approfondies afin d'appréhender au mieux le risque suicidaire chez les gais et les lesbiennes, celui-ci étant dépendant de la définition qui est donnée à l'homosexualité. Certaines recherches mettent en lien la sursuicidalité des homosexuels avec la divulgation de leur homosexualité. Selon D'Augelli et al. [24], les jeunes homosexuels ayant annoncé leur homosexualité auraient quatre fois plus de risque de tenter de se suicider que ceux n'ayant pas divulgué leur orientation sexuelle. Le risque suicidaire au moment de l'annonce serait d'autant plus élevé lorsque cette divulgation est réalisée à un âge précoce, certains jeunes n'ayant pas les ressources suffisantes pour faire face à un éventuel rejet [25]. Cette recherche vise à appréhender les liens existants entre le risque suicidaire des jeunes homosexuels et la divulgation de leur homosexualité.

L'annonce de son homosexualité constitue une situation stressante, marquée par la peur du rejet et des réactions négatives. Cette divulgation peut générer un important mal-être, le jeune homosexuel s'exposant au jugement de l'autre et à un éventuel rejet. Mais elle représente également une étape importante dans la construction de l'identité homosexuelle. Libérés du poids du secret, les jeunes homosexuels peuvent alors ressentir un important sentiment de libération, de soulagement, suite à cette divulgation. C'est de ce paradoxe, entre souffrance et libération, qu'est née cette recherche. Alors que la littérature se centre sur les liens existants entre le mal-être des jeunes homosexuels et les réactions de leur entourage, nous avons fait le 
choix de nous intéresser à la manière dont les jeunes homosexuels font face au stress de cette divulgation, axe peu étudié jusqu'ici. Cette recherche se propose donc d'étudier les stratégies que les jeunes homosexuels mettent en place lors de l'annonce de leur homosexualité. Notre objectif final étant de proposer des pistes d'accompagnement adaptées aux difficultés particulières que ces jeunes rencontrent lors de la divulgation de leur homosexualité.

\section{Méthode}

\section{- Participants}

Cette étude a été menée auprès de 200 jeunes se disant homosexuels (69 femmes et 131 hommes) qui ont vécu au moins une annonce de leur homosexualité qu'ils ont vécu comme stressante. L'âge des participants est compris entre 16 et 25 ans $(\mathrm{M}=21,7$; ET =2,6), Au début du questionnaire, il a été précisé aux participants qu'ils devaient répondre à l'ensemble des questions en se basant sur la divulgation de leur homosexualité qui a été la plus stressante au cours de leur vie. Ce critère a été renforcé par une question placée au milieu du questionnaire: "Évaluez le niveau de stress de cette situation en comparaison avec les autres coming-out dont vous avez fait l'expérience durant votre vie (s'il y en a eu d'autres) ». Les réponses allant de 0 (cette situation était parmi les moins stressantes que j'ai jamais vécues) à 4 (cette situation était parmi les plus stressantes que j'ai jamais vécues). Seuls les questionnaires dont la réponse a été « 4 » ont été retenus.

\section{- Passation}

Les sujets ont été rencontrés par le biais d'internet. Dans divers sites de rencontres, tous les profils de personnes se disant homosexuels et ayant entre 18 et 25 ans ont été retenus, sans autre critère de sélection. Un mail a été envoyé à l'ensemble de ces profils, explicitant l'étude, sa procédure, ses objectifs et précisant les coordonnées des personnes à contacter pour y participer. Les personnes intéressées nous ont contactés et ont été informées plus en détails de la recherche. Après avoir répondu à leurs interrogations, un questionnaire d'une durée approximative de 30 à 45 minutes leur a été envoyé. Les participants y ont répondu anonymement et gratuitement, après avoir signé un formulaire de consentement. Un retour des résultats leur a été envoyé une fois les premières analyses réalisées. 


\section{- Stratégies de coping}

La version française de la «Way of Coping Check-list» [26] de Folkman et Lazarus (1988) [27] a été administrée aux participants. Cette échelle composée de 68 items évalue les stratégies de coping mises en œuvre lors d'un évènement stressant. En se basant sur la divulgation de leur homosexualité la plus stressante, les sujets ont répondu aux items sur une échelle Likert allant de $0:$ :pas du tout» à 4: "beaucoup ». Une analyse factorielle à composante principale avec rotation (Varimax) a été réalisée. Nous avons fait le choix de retenir huit facteurs qui expliquent $40,7 \%$ de la variance (tableau 1).

\section{Placer le tableau 1 ici}

\section{- Évaluation de la situation}

La préparation, la provocation et la répétition de l'annonce de leur homosexualité ont été évaluées. Les participants ont répondu aux questions suivantes "l'avez-vous prévu?», «l'avez-vous provoqué? » sur une échelle Likert allant de 0 : «pas du tout » à $4:$ : tout à fait». Ils ont déterminé le nombre d'annonces de leur homosexualité qu'ils ont vécu de manière stressantes au cours de leur vie. Quatre modalités de réponses étaient proposées : " un », " deux ou trois », " trois ou quatre », " plus de quatre ». Le questionnaire débute par une question ouverte demandant aux participants de décrire la divulgation de leur homosexualité la plus stressante qu'ils ont vécu afin qu'ils se replongent dans la situation.

\section{- Idéations et tentatives de suicide}

La présence d'idées suicidaires et de tentatives de suicide a été évalué à l'aide de deux items dichotomiques : "Au cours de mon coming-out le plus stressant, j'ai tenté de mettre fin à mes jours? ", "Au cours de mon coming-out le plus stressant, j'ai pensé à mettre fin à mes jours ? ». Pour chaque item, deux modalités de réponses ont été proposées aux participants: « Oui » et « Non» cotées respectivement 1 et 0 .

\section{Résultats}




\section{- Caractéristiques de la situation stressante}

$\mathrm{Au}$ sein de notre échantillon, l'annonce la plus stressante a eu lieu en moyenne à 18 ans $(\mathrm{ET}=2,5)$, c'est-à-dire il y a 3,5 ans $(\mathrm{ET}=2,4)$. Pour la plupart, l'annonce la plus stressante a été celle réalisée auprès de leur famille. Que ce soit auprès des parents $(\mathrm{N}=127)$, auprès des parents et d'autres membres de leur famille $(\mathrm{N}=25)$ ou encore seulement à d'autres membres de leur famille $(\mathrm{N}=10)$. Pour trente cinq répondants, la divulgation la plus stressante a été celle réalisée à un de leur ami. Rares sont ceux qui évoquent des annonces dans le cadre professionnel $(\mathrm{N}=3)$ ou auprès d'un spécialiste $(\mathrm{N}=1)$.

\section{- Les stratégies de coping}

Les stratégies utilisées par l'ensemble de notre échantillon sont très hétérogènes (tableau 2). Les deux sexes utilisent des stratégies similaires sauf pour les comportements addictifs (F6) $\left(t_{\text {femmes/hommes (198) }}=2,1 ; \mathrm{p}=0,03\right)$ et la confrontation aux autres $(\mathrm{F} 8)\left(\mathrm{t}_{\text {femmes/hommes (198) }}=\right.$ $2,9 ; \mathrm{p}=0,004)$.

\section{Placer le tableau 2 ici}

Certains facteurs présentent des intercorrélations (tableau 3). Les corrélations entre la culpabilisation, l'isolement, les conduites suicidaires (F1), la fuite et l'évitement (F3) mais aussi les comportements addictifs (F6) sont particulièrement fortes.

\section{Placer le tableau 3 ici}

\section{- Idéations, conduites suicidaires et stratégies de coping}

Au sein de notre échantillon, $38 \%$ des participants ont déclaré avoir pensé à mettre fin à leurs jours au cours de la divulgation de leur homosexualité la plus stressante et $23 \%$ sont passés à l'acte. Aucune différence n'a été constatée pour les hommes et les femmes. Les participants qui ont eu des idéations suicidaires (tableau 4) ou des conduites suicidaires (tableau 5) n'ont pas employé les mêmes stratégies de coping que ceux qui n'en ont pas eu.

\section{Placer le tableau 4 ici}




\section{Placer le tableau 5 ici}

\section{- Préparation, provocation, répétition et stratégies de coping}

Dans cette étude, 46,5\% des participants ont « un peu », voire " pas du tout » prévu cette annonce $(\mathrm{M}=1,9, \mathrm{ET}=1,6), 39,5 \%$ affirment l'avoir " un peu », voire "pas du tout » provoquée $(\mathrm{M}=1,6, \mathrm{ET}=1,6)$. Au sein de notre échantillon, seulement $11 \%$ déclarent ne s'être sentis qu'une seule fois stressés par l'annonce de leur homosexualité, alors que $36 \%$ ont été stressés deux à trois fois et 53\% ont rencontré plus de quatre annonces stressantes. La préparation, la provocation et la répétition des divulgations stressantes sont étroitement liées à certaines stratégies de coping (tableau 6).

\section{Placer le tableau 6 ici}

\section{IV.Discussion}

Dans notre échantillon, 161 participants ont déclaré s'être sentis « mieux à la fin de leur coming-out qu'au début » (item 30). De plus, 176 répondants ont affirmé que cette expérience leur a permis «de changer et d'évoluer en bien » (item 23). Malgré le stress, cette annonce constitue donc une étape importante, comme le précise Iwasaki et Ristock [3], elle fait partie intégrante d'un processus de « reconnaissance de soi », favorisant le développement personnel et le bien-être [6]. La «demande de soutien social» est le facteur le plus utilisé par les participants. Lors de cette annonce, les homosexuels ont tendance à faire appel à leurs amis, sollicitant leur aide et leurs conseils, 141 sujets déclarent « avoir parlé à quelqu'un pour en savoir plus sur la situation » (item 8). Ce type de stratégies est utilisé par l'ensemble de notre échantillon, que les participants aient présenté ou non des conduites suicidaires. Nous pouvons penser que ceux qui ont réalisé une tentative de suicide n'ont pas trouvé le soutien qu'ils recherchaient, alors que les jeunes qui ne sont pas passés à l'acte ont bénéficié de personnes les soutenant. Ces résultats vont dans le sens des conclusions d'Eisenberg et Resnick [28] qui attestent que lors de l'annonce de son homosexualité, un important soutien serait un bon facteur de protection contre les risques suicidaires. A contrario, son absence, serait un bon prédicteur pour les tentatives de suicide [29]. Les homosexuels ont rarement recours à des professionnels pour les aider à faire face à la divulgation de leur homosexualité, 
166 participants affirment ne pas avoir du tout sollicité un spécialiste (item 22). Cette annonce les confronte à l'inconnu, 144 sujets affirment ne pas avoir du tout pu faire appel à une situation passée, à leur expérience (item 49); 106 ont déclaré ne pas du tout savoir ce qu'il fallait faire (item 50) et 158 jeunes n'ont pas pensé à ce que ferait une personne qu'ils admirent (item 64). Notre société contient peu de modèles homosexuels socialement valorisés, rares sont les jeunes homosexuels qui parviennent à s'appuyer sur une personne qu'ils admirent pour trouver des solutions. La plupart des groupes minoritaires partagent cette appartenance avec leur famille et y trouvent des ressources les protégeant de la stigmatisation [6]. Pour les homosexuels, leur orientation sexuelle constitue plutôt un élément de rejet [2, 30, 31]. Ainsi, seul le groupe de pairs semble faire office de soutien et de modèle. Au cours de leur vie, les homosexuels sont amenés à divulguer leur homosexualité à plusieurs reprises, l'annonce la plus stressante étant souvent celle réalisée auprès de la famille. Nos résultats démontrent que plus les homosexuels vont vivre des divulgations qu'ils jugent stressantes, plus ils vont avoir recours à des stratégies d'isolement, de culpabilité, aux conduites addictives et suicidaires. Ces modes de coping peuvent présenter des bénéfices à court terme mais sont plutôt préjudiciables sur le long terme. Mais la répétition des annonces stressantes est aussi liée aux stratégies de résolution de problème. Ainsi, elle favorise aussi le recours à des stratégies réflectives et leur permet ainsi d'élaborer des plans d'actions de plus en plus efficients. Les homosexuels qui ont présenté des ideations suicidaires lors de la divulgation de leur homosexualité ont eu beaucoup recours à la fuite, à l'isolement, aux conduites addictives et très peu aux stratégies d'évolution personnelle. Ils ne sont pas parvenus à réévaluer de manière positive la situation et à tirer des bénéfices de cette annonce. Ainsi, pour ces jeunes, l'annonce de leur homosexualité n'a pas été une étape favorisant leur bien-être, mais a plutôt représenté une situation de grande souffrance. Parmi nos répondants, seulement $23,5 \%$ ont tout à fait prévu la divulgation de leur homosexualité et $36,5 \%$ l'ont tout à fait provoqué. Cette annonce est souvent faite dans la précipitation, sans faire l'objet d'une préparation, elle peut être imposée par l'entourage ou par la situation. Nos résultats attestent que la préparation et l'initiative favorisent les stratégies d'analyse, la recherche de solutions, l'évaluation positive de la situation et donc une meilleure gestion de cette situation stressante. Ces résultats rendent compte de la nécessité d'accompagner ces jeunes dans cette annonce.

A partir des résultats de cette recherche, nous avons dégagé des pistes d'accompagnement sur le plan cognitif, émotionnel et comportemental. La prise en charge proposée s'inspire des 
programmes de gestion du stress [32]. Tout d'abord, les cognitions associées à cette annonce doivent être repérées, tout comme les croyances irrationnelles, les attentes et les objectifs du patient [33]. Les avantages et les inconvénients à court, moyen et long terme doivent être évalués, ainsi que les conséquences de cette annonce. Ce travail cognitif favorisera l'anticipation de la divulgation de leur homosexualité, mais aussi des réactions et des conséquences auxquelles le jeune peut se confronter. Des techniques de restructuration cognitive telles le scénario catastrophe, l'acceptation des conduites de rejet, ou la technique de la flèche descendante peuvent être utilisées. La peur d'être submergé par leurs émotions conduit les jeunes homosexuels à vouloir les contrôler. Un travail centré sur les émotions peut leur être proposé. Les accompagner dans l'identification et l'évaluation de leurs émotions favoriserait l'acceptation de leur vécu émotionnel et atténuerait leur désir de contrôle, la première étape pour gérer et accepter les émotions étant de les connaitre [34]. En parallèle, des techniques de respiration et de relaxation peuvent être utilisées pour apprendre au patient à se détendre et à mieux faire face aux évènements stressants [35, 36]. Des techniques comportementales peuvent être employées pour finaliser la préparation. Elles peuvent prendre la forme d'une exposition en jeu de rôle avec le professionnel. Cette technique permettra au jeune d'analyser ses émotions et ses cognitions lors de la mise en situation et d'améliorer sa communication. De nombreux jeunes homosexuels recherchent des conseils et de l'aide lors de l'annonce de leur homosexualité. Déterminer toutes les personnes susceptibles de l'aider, et l'inciter à les solliciter, aura une fonction de réassurance et favorisera la diminution des stratégies d'isolement et de mise à distance. Le professionnel se doit de constituer un soutien sur lequel le jeune peut s'appuyer. La plupart des jeunes interrogés nous ont déclaré avoir une vision négative de la psychologie et de la psychiatrie qu'ils assimilent à des disciplines homophobes. Nos résultats démontrent que rares sont ceux qui font appel à un spécialiste lors de leur divulgation la plus stressante. Comme le précise l'«American Psychological Association » [37], un important effort doit être fait dans la formation des professionnels, notamment concernant les difficultés que les homosexuels rencontrent de par leur appartenance à une minorité sexuelle.

Cette recherche se limite à la divulgation la plus stressante vécue par ces jeunes. Il serait intéressant d'approfondir ces données en étudiant la manière dont les jeunes homosexuels font face aux autres annonces de leur homosexualité, mais aussi à d'autres évènements stressants, afin de dégager les spécificités de cette situation. Ces dimensions mériteraient de faire l'objet 
d'une seconde étude. Le recrutement par internet implique un certain nombre de biais, les participants ayant plus de possibilités de mentir lors de la passation. Cependant, les différents échanges que nous avons réalisés avec les participants et la longueur du questionnaire (30 à 45 minutes) rendent compte de leur motivation. Cette recherche constitue une étude exploratoire, les résultats obtenus seront appuyés ou contredits par une méthodologie qualitative qui est actuellement en cours de réalisation.

\section{Conclusion}

$\mathrm{Au}$ cours de la divulgation de leur homosexualité, les stratégies de culpabilité, d'isolement, de fuite se mêlent aux stratégies d'évolution personnelle, de demande de soutien et d'affirmation de soi. Lorsque cette annonce est préparée et est à l'initiative du jeune, elle favorise l'évolution personnelle et une meilleure gestion du stress. À l'inverse, lorsque l'annonce de son homosexualité est peu préparée, les stratégies de culpabilisation, les conduites addictives et suicidaires sont privilégiées. Ces résultats démontrent la nécessité de préparer cette annonce et de sensibiliser les professionnels afin qu'ils soient à même d'accompagner les jeunes homosexuels dans cette démarche. Un travail cognitif, comportemental et émotionnel s'inspirant des programmes de gestion du stress nous apparaît pertinent. Cette annonce étant souvent l'occasion de passage à l'acte suicidaire, ces pistes d'accompagnement issues de cette recherche constituent un outil de prévention du suicide chez les jeunes homosexuels au cours de la divulgation de leur homosexualité.

\section{Bibliographie}

[1] Savin-Williams RC. Mom, Dad, I'm gay: How families negotiate coming out. Washington, DC: American Psychological Association; 2001.

[2] Willoughby BLB, Malik NM, Lindahl KM. Parental Reactions to Their Sons' Sexual Orientation Disclosures: The Roles of Family Cohesion, Adaptability, and Parenting Style. Psychol Men Masc 2006;7(1):14-26.

[3] Iwasaki Y, Ristock JL. The nature of stress experienced by lesbians and gay men. Anxiety Stress Coping 2007;20(3):299-319.

[4] Coursaud JB. L'homosexualité entre préjugés et réalités. Toulouse: Milan; 2002. 
[5] Riley BH. GLB Adolescent's "Coming-out". J Child Adolesc Psychiatr Nurs 2010;23:310 .

[6] Julien D. Famille d'origine et homosexualité. In: Simard M, Alary J, editors. Comprendre la famille : actes du 5e symposium québécois de recherche sur la famille. Québec: Presse Universitaire du Québec; 2000. p. 210-22.

[7] Lewis RJ, Derlega VJ, Griffin JL, Krowinski AC. Stressors for Gay Men and Lesbians: Life Stress, Gay-Related Stress, Stigma Consciousness, and Depressive Symptoms. J Soc Clin Psychol 2003;22(6):716-29.

[8] D’Augelli AR. Mental Health Problems among Lesbian, Gay, and Bisexual Youths Ages 14 to 21. Clin Child Psychol Psychiatry 2002;7(3):433-56

[9] Meyer IH. Prejudice, social stress, and mental health in lesbian, gay, and bisexual populations: conceptual issues and research evidence. Psychol Bull 2003;129(5):674-97.

[10] Robohm JS, Litzenberger BW, Pearlman LA. Sexual abuse in lesbian and bisexual young women: Associations with emotional/behavioral difficulties, feelings about sexuality, and the "coming out" process. J Lesbian Stud 2003;7(4):31-47.

[11] Meyer IH. Minority stress and mental health in gay men. J Health Soc Behav 1995;36(1):38-56.

[12] Lazarus RS, Folkman S. Stress, Appraisal and Coping. New York: Springer Publishing; 1984.

[13] Lazarus RS, Folkman S. Transactional theory and research on emotions and coping. European Journal of Personality 1987;1(3):141-69.

[14] Lazarus RS, Folkman S. Transactional theory and research on emotions and coping. Eur J Pers 1987;1(3):141-69.

[15] D'Augelli AR, Hershberger SL, Pilkington NW. Suicidality patterns and sexual orientation-related factors among lesbian, gay, and bisexual youths. Suicide Life Threat Behav 2001;31(3):250-64.

[16] Garofalo R, Wolf RC, Kessel S, Palfrey SJ, DuRant RH. The association between health risk behaviors and sexual orientation among a school-based sample of adolescents. Pediatrics 1998;101(5):895-902.

[17] Garofalo R, Wolf RC, Wissow LS, Woods ER, Goodman E. Sexual orientation and risk of suicide attempts among a representative sample of youth. Arch Pediatr Adolesc Med 1999;153(5):487-93. 
[18] Russell ST, Joyner K. Adolescent sexual orientation and suicide risk: evidence from a national study. Am J Public Health 2001;91(8):1276-81.

[19] Saewyc EM, Skay CL, Hynds P, Pettingell S, Bearinger LH, Resnick MD, et al. Suicidal ideation and attempts in North American school-based surveys: are bisexual youth at increasing risk? J LGBT Health Res 2007;3(2):25-36.

[20] Silenzio VM, Pena JB, Duberstein PR, Cerel J, Knox KL. Sexual orientation and risk factors for suicidal ideation and suicide attempts among adolescents and young adults. Am J Public Health 2007;97(11):2017-9.

[21] Suicide Prevention Resource Center. Suicide risk and prevention for lesbian, gay, bisexual, and transgender youth. M.A. Newton: Education Development Center Inc; 2008.

[22] Beck F, Guilbert P, Gauthier A. Baromètre santé 2005. Attitudes et comportements de santé. Saint Denis: INPES; 2007.

[23] Lhomond B, Saurel-Cubizolles MJ. Orientation sexuelle, violences contre les femmes et santé. Résultats de l'enquête nationale sur les violences envers les femmes en France. In: Broqua C, Lert F, Souteyrand Y, editors. Homosexualités au temps du sida. Paris: ANRS; 2003. p. 107-30.

[24] D'Augelli AR, Hershberger SL, Pilkington NW. Lesbian, gay, and bisexual youth and their families: disclosure of sexual orientation and its consequences. Am J Orthopsychiatry 1998;68(3):361-75.

[25] Remafedi G, Farrow JA, Deisher RW. Risk factors for attempted suicide in gay and bisexual youth. Pediatrics 1991;87(6):869-75.

[26] Graziani P, Rusinek S, Servant D, Hautekeete-Sence D, Hautekeete M. Validation française du questionnaire de coping « way of coping check-list-revised » (W.C.C.-R.) et analyse des événements stressants du quotidien. J Ther Comport Cogn 1998;8(3):100-12.

[27] Folkman S, Lazarus RS. Ways of Coping Questionnaire : Research edition. Palo Alto: Consulting Psychologists Press; 1988.

[28] Eisenberg ME, Resnick MD. Suicidality among gay, lesbian and bisexual youth: the role of protective factors. J Adolesc Health 2006;39(5):662-8.

[29] Safren SA, Heimberg RG. Depression, hopelessness, suicidality, and related factors in sexual minority and heterosexual adolescents. J Consult Clin Psychol 1999;67(6):859-66.

[30] Pilkington NW, D'Augelli AR. Victimization of lesbian, gay, and bisexual youth in community settings. J Community Psychol 1995;23(1):34-56.

[31] SOS Homophobie. Rapport 2004 sur l'homophobie. Paris: SOS Homophobie; 2004. 
[32] Cottraux J. Les thérapies comportementale et cognitives. Issy-les-Moulineaux: Elsevier Masson; 2004.

[33] Millêtre B. Application des tcc au processus de «coming-out » chez une patiente homosexuelle. J Ther Comport Cogn 2004;14(3):141-45.

[34] Ravon D. Apprivoiser ses émotions - L'intelligence des situations. Paris: Eyrolles; 2008.

[35] Jacobson E. Savoir relaxer pour combattre le stress. Montréal: Editions de l'Homme 1980.

[36] Schultz JH. Manuel pratique du training autogène. Paris: Presses Universitaires de France; 1965.

[37] American Psychological Association. Guidelines for psychotherapy with lesbian, gay, and bisexual clients. Washington, DC: American Psychological Association; 2000.

\section{Conflit d'intérêt : aucun}

Tableau 1 : Caractéristiques des différents facteurs de coping

\begin{tabular}{|l|c|c|}
\hline \multicolumn{1}{|c|}{ Facteurs de coping } & $\begin{array}{c}\text { Nombre } \\
\text { d'items }\end{array}$ & $\begin{array}{c}\text { Variance } \\
\text { expliquée }\end{array}$ \\
\hline $\begin{array}{l}\text { Facteur 1 (F1) : « culpabilisation, isolement et conduites } \\
\text { suicidaires » }\end{array}$ & 16 & $8,8 \%$ \\
\hline $\begin{array}{l}\text { Facteur 2 (F2) : « évolution personnelle et réévaluation } \\
\text { positive » }\end{array}$ & 8 & $6,6 \%$ \\
\hline Facteur 3 (F3) : « fuite et évitement» & 4 & $5,2 \%$ \\
\hline Facteur 4 (F4) : « demande de soutien social» & 5 & $4,6 \%$ \\
\hline Facteur 5 (F5) : « résolution de problème » & 5 & $4,1 \%$ \\
\hline Facteur 6 (F6) : « comportement addictif» & 4 & $3,9 \%$ \\
\hline Facteur 7 (F7) : « contrôle des émotions » & 4 & $3,8 \%$ \\
\hline Facteur 8 (F8) : « confrontation aux autres » & 3 & $3,7 \%$ \\
\hline
\end{tabular}

Tableau 2 : Moyennes et écarts types par ordre décroissant aux différents facteurs de coping utilisés par les jeunes homosexuels au cours de leur coming-out le plus stressant (échelle Likert 0-4)

\begin{tabular}{|l|c|c|}
\hline \multicolumn{1}{|c|}{ Facteurs de coping } & Moyennes & Ecarts-type \\
\hline F2: « évolution personnelle et réévaluation positive » & 1,6 & 0,7 \\
\hline F4 : « demande de soutien social » & 1,6 & 0,8 \\
\hline F5 : « résolution de problème » & 1,4 & 0,6 \\
\hline F7 : « contrôle des émotions » & 1,2 & 0,7 \\
\hline
\end{tabular}


F3 : « fuite et évitement »

F1 : «culpabilisation, isolement et conduites suicidaires »

F8 : « confrontation aux autres »

F6 : « comportement addictif»

\begin{tabular}{|c|c|}
\hline 1 & 0,9 \\
\hline 0,9 & 0,6 \\
\hline 0,6 & 0,6 \\
\hline 0,6 & 0,8 \\
\hline
\end{tabular}

Tableau 3 : Corrélations de Bravais Pearson entre les différents facteurs de coping

\begin{tabular}{|l|l|l|l|l|l|l|l|l|}
\hline & F1 & F2 & F3 & F4 & F5 & F6 & F7 & F 8 \\
\hline F1 & 1 & & & & & & & \\
\hline F2 & $\mathbf{- 0 , 1 5 *}$ & 1 & & & & & & \\
\hline F3 & $\mathbf{0 , 4 2 * *}$ & NS & 1 & & & & & \\
\hline F4 & NS & $\mathbf{0 , 3 2 * *}$ & NS & 1 & & & & \\
\hline F5 & $\mathbf{0 , 1 9 * *}$ & $\mathbf{0 , 3} * *$ & NS & $\mathbf{0 , 2}$ (1* $^{* *}$ & 1 & & & \\
\hline F6 & $\mathbf{0 , 4 4 * *}$ & NS & $\mathbf{0 , 1 9 * *}$ & NS & NS & 1 & & \\
\hline F7 & $\mathbf{0 , 1 6 *}$ & NS & $\mathbf{0 , 1 7 *}$ & NS & $\mathbf{0 , 1 7 *}$ & $\mathbf{0 , 1 6}^{*}$ & 1 & \\
\hline F8 & $\mathbf{0 , 2 4 * *}$ & $\mathbf{0 , 1 9 * *}$ & $\mathbf{0 , 1 7 *}$ & $\mathbf{0 , 2} \mathbf{2}^{* *}$ & NS & $\mathbf{0 , 2 5}^{* *}$ & $\mathbf{0 , 1 5 *}$ & 1 \\
\hline
\end{tabular}

$* p \leq 0,05$

$* * p \leq 0,01$

NS: non significatif

$F 1=$ « culpabilisation, isolement et conduites suicidaires »

$F 2=$ "évolution personnelle et réévaluation positive»

$F 3=$ "fuite et évitement »

$F 4=$ « demande de soutien social »

$F 5=$ " résolution de problème »

$F 6=$ " comportement addictif »

$F 7=$ « contrôle des émotions »

F8 = « confrontation aux autres »

Tableau 4 : Moyennes (écarts types) et comparaisons (t-Student) entre les scores aux différents facteurs de coping des jeunes homosexuels selon la présence d'idées suicidaires

\begin{tabular}{|l|c|c|c|c|}
\hline \multicolumn{1}{|c|}{ Facteurs de coping } & \multicolumn{3}{c|}{ Présence d'idées suicidaires } \\
\hline & $\begin{array}{c}\text { Non } \\
(\mathrm{n}=124)\end{array}$ & $\begin{array}{c}\text { Oui } \\
(\mathrm{n}=76)\end{array}$ & $\mathrm{t}_{(198)}$ & $\mathrm{p}$ \\
\hline $\begin{array}{l}\text { F1 : « culpabilisation, isolement et conduites } \\
\text { suicidaires » }\end{array}$ & $\mathbf{0 , 6}(\mathbf{0 , 4})$ & $\mathbf{1 , 5}(\mathbf{0 , 6})$ & $\mathbf{- 1 3 , 3}$ & $\mathbf{0 , 0 0 0 1}$ \\
\hline F2: « évolution personnelle et réévaluation positive » & $\mathbf{1 , 7 ( 0 , 7 )}$ & $\mathbf{1 , 4}(\mathbf{0 , 7 )}$ & $\mathbf{2 , 4}$ & $\mathbf{0 , 0 1 6}$ \\
\hline F3 : « fuite et évitement » & $\mathbf{0 , 8}(\mathbf{0 , 7})$ & $\mathbf{1 , 4}(\mathbf{1 , 2})$ & $\mathbf{- 4}$ & $\mathbf{0 , 0 0 0 1}$ \\
\hline F4 : « demande de soutien social » & $1,6(0,8)$ & $1,6(0,8)$ & $-0,3$ & $\mathrm{NS}$ \\
\hline F5 : « résolution de problème » & $1,3(0,6)$ & $1,5(0,6)$ & $-1,8$ & $\mathrm{NS}$ \\
\hline F6 : « comportement addictif» & $\mathbf{0 , 4}(\mathbf{0 , 6})$ & $\mathbf{0 , 9}(\mathbf{0 , 9})$ & $\mathbf{- 4 , 9}$ & $\mathbf{0 , 0 0 0 1}$ \\
\hline F7 : « contrôle des émotions » & $1,2(0,7)$ & $1,3(0,6)$ & $-0,7$ & $\mathrm{NS}$ \\
\hline F8 : « confrontation aux autres » & $\mathbf{0 , 6}(\mathbf{0 , 5})$ & $\mathbf{0 , 7}(\mathbf{0 , 7})$ & $\mathbf{- 2}$ & $\mathbf{0 , 0 5}$ \\
\hline
\end{tabular}


Tableau 5: Moyennes, écarts types et comparaisons (t-Student) entre les scores aux différents facteurs de coping des jeunes homosexuels selon la présence de conduite suicidaire

\begin{tabular}{|l|c|c|c|c|}
\hline \multicolumn{1}{|c|}{ Facteurs de coping } & \multicolumn{3}{|c|}{ Réalisation d'une tentative de suicide } \\
\hline & $\begin{array}{c}\text { Non } \\
(\mathrm{n}=154)\end{array}$ & $\begin{array}{c}\text { Oui } \\
(\mathrm{n}=46)\end{array}$ & $\mathrm{t}_{(198)}$ & $\mathrm{p}$ \\
\hline $\begin{array}{l}\text { F1 : « culpabilisation, isolement et conduites } \\
\text { suicidaires » }\end{array}$ & $\mathbf{0 , 7}(\mathbf{0 , 4})$ & $\mathbf{1 , 7}(\mathbf{0 , 6})$ & $\mathbf{- 1 2 , 2}$ & $\mathbf{0 , 0 0 0 1}$ \\
\hline F2: « évolution personnelle et réévaluation positive » & $1,6(0,7)$ & $1,4(0,6)$ & 1,5 & $\mathrm{NS}$ \\
\hline F3 : « fuite et évitement » & $\mathbf{0 , 9}(\mathbf{1})$ & $\mathbf{1 , 4}(\mathbf{0 , 8})$ & $\mathbf{- 2 , 7}$ & $\mathbf{0 , 0 0 7}$ \\
\hline F4 : « demande de soutien social» & $1,6(0,8)$ & $1,5(0,8)$ & 2,9 & $\mathrm{NS}$ \\
\hline F5 : « résolution de problème » & $1,4(0,6)$ & $1,4(0,7)$ & $-0,3$ & $\mathrm{NS}$ \\
\hline F6 : « comportement addictif » & $\mathbf{0 , 5}(\mathbf{0 , 6})$ & $\mathbf{1 , 1}(\mathbf{0 , 9})$ & $\mathbf{- 5 , 1}$ & $\mathbf{0 , 0 0 0 1}$ \\
\hline F7 : « contrôle des émotions » & $1,3(0,7)$ & $1,3(0,7)$ & $-0,5$ & $\mathrm{NS}$ \\
\hline F8 : « confrontation aux autres » & $0,6(0,6)$ & $0,7(0,6)$ & $-0,8$ & $\mathrm{NS}$ \\
\hline
\end{tabular}

Tableau 6 : Corrélation de Bravais Pearson entre la préparation, la provocation, le nombre de coming-out stressants, et les facteurs de coping

\begin{tabular}{|l|c|c|c|}
\hline Facteurs de coping & Préparation & Provocation & $\begin{array}{c}\text { Nombre de coming-out } \\
\text { stressants }\end{array}$ \\
\hline F1 : « culpabilisation, isolement et & $\begin{array}{c}\mathrm{r}=-0,035 \\
\mathrm{p}=\mathrm{NS}\end{array}$ & $\begin{array}{c}\mathrm{r}=-0,12 \\
\mathrm{p}=\mathrm{NS}\end{array}$ & $\begin{array}{c}\mathbf{r}=\mathbf{0 , 3 2} \\
\mathbf{p}=\mathbf{0 , 0 0 0 1}\end{array}$ \\
\hline Fonduites suicidaires » & $\mathbf{r}=\mathbf{0 , 2 2}$ & $\mathbf{r}=\mathbf{0 , 1 6}$ & $\mathrm{p}=-0,06$ \\
réévaluation positive » & $\mathbf{p}=\mathbf{0 , 0 0 2}$ & $\mathrm{p}=\mathbf{0 , 0 2}$ & $\mathrm{p}=\mathrm{NS}$ \\
\hline F3 : « fuite et évitement» & $\mathrm{r}=0,22$ & $\mathrm{r}=-0,08$ & $\mathrm{r}=0,13$ \\
& $\mathrm{p}=\mathrm{NS}$ & $\mathrm{p}=\mathrm{NS}$ & $\mathrm{p}=\mathrm{NS}$ \\
\hline F4 : « demande de soutien social » & $\mathbf{r}=\mathbf{0 , 1 4}$ & $\mathrm{r}=0,03$ & $\mathrm{r}=0,04$ \\
& $\mathbf{p}=\mathbf{0 , 0 5}$ & $\mathrm{p}=\mathrm{NS}$ & $\mathrm{p}=\mathrm{NS}$ \\
\hline F5 : « résolution de problème » & $\mathbf{r}=\mathbf{0 , 3 9}$ & $\mathbf{r}=\mathbf{0 , 2 2}$ & $\mathbf{r}=\mathbf{0 , 1 7}$ \\
& $\mathbf{p}=\mathbf{0 , 0 0 0 1}$ & $\mathbf{p}=\mathbf{0 , 0 0 2}$ & $\mathbf{p}=\mathbf{0 , 0 2}$ \\
\hline F6 : « comportement addictif» & $\mathrm{r}=-0,12$ & $\mathrm{r}=-0,03$ & $\mathbf{r}=\mathbf{0 , 2 5}$ \\
& $\mathrm{p}=\mathrm{NS}$ & $\mathrm{p}=\mathrm{NS}$ & $\mathbf{p}=\mathbf{0 , 0 0 1}$ \\
\hline F7 : « contrôle des émotions » & $\mathrm{r}=0,01$ & $\mathrm{r}=-0,02$ & $\mathrm{r}=0,06$ \\
& $\mathrm{p}=\mathrm{NS}$ & $\mathrm{p}=\mathrm{NS}$ & $\mathrm{p}=\mathrm{NS}$ \\
\hline
\end{tabular}

